\title{
Zamorano y sus Contribuciones a la Agricultura Sostenible a Través del Control Biológico de Plagas
}

\author{
Ronald D. Cave ${ }^{1}$, Rogelio Trabanino ${ }^{2}$ y Abelino Pitty ${ }^{3}$
}

Resumen. El Centro para el Control Biológico en Centro América (CCBCA) fue creado en 1989 por la Escuela Agrícola Panamericana, financiado por la United States Agency for International Development (USAID) en Honduras. El CCBCA se enfocó en el control biológico clásico, el aumento y la conservación de enemigos naturales. En el 2000 cambió el nombre de Laboratorio de Control Biológico y se enfocó en el aprender haciendo de los estudiantes de la Escuela Agrícola Panamericana y la producción y comercialización de enemigos naturales de plagas agrícolas. El único parasitoide conocido que ataca al picudo de las bromelias (Metamasius quadrilineatus), fue encontrado en las investigaciones del CCBCA; resultó una especie nueva nombrada Lixadmontia franki que fue liberada en Florida en el 2007 para el control biológico del picudo mejicano de las bromelias (Metamasius callizona). Se determinaron los organismos parasíticos de las plagas Plutella xylostella, Mocis latipes, Spodoptera frugiperda, Leptophobia aripa y Liriomyza spp. Cuatro especies de avispas parasíticas exóticas, un baculovirus, un hongo y tres picudos fueron introducidos a Honduras para control biológico clásico: Cotesia plutellae (parasitoide asiático de P. xylostella); Diadromus collaris (parasitoide pupal de P. xylostella); Telenomus remus (parasitoide que ataca a los huevos de 30 especies de lepidópteros); una especie de Eretmocerus (originaria de India, para controlar Bemisia tabaci); los picudos Neochetina bruchi y Neochetina eichhorniae y el hongo Cercospora piaropi (para el control de la maleza acuática lirio de agua (Eichhornia crassipes); y el picudo Neohydronomous affinis (controlador biológico de la maleza acuática lechuga de agua (Pistia stratiotes). En el 2000, Zamorano cambió la estrategia del uso de control biológico y empezó a incursionar en la producción comercial de microrganismos para el control de plagas, debido a una demanda no satisfecha. Los controladores biológicos que ha producido son el hongo antagonista Trichoderma harzianum (Trichozam ${ }^{\mathrm{TM}}$ ) para combatir hongos en el suelo, Beauveria bassiana $\left(\right.$ Bazam $\left.^{\mathrm{TM}}\right)$ para controlar lepidópteros y coleópteros, Lecanicillium lecanii $\left(\right.$ Verzam $^{\mathrm{TM}}$ ) para el control de áfidos y mosca blanca, Metarhizium anisopliae (Metazam ${ }^{\mathrm{TM}}$ ) para el control de salivazo (Aeneolamia spp.) y larvas de coleópteros en caña de azúcar, y Purpureocillium lilacinum (Pazam ${ }^{\mathrm{TM}}$ ) para controlar nematodos. Además, ha reproducido y vendido la chinche depredadora Orius insidiosus para el control de trips, áfidos y mosca blanca, el ácaro depredador Neoseiulus longispinosus para el control de la arañita roja (Tetranychus spp.) y el nematodo entomófago Heterorhabditis bacteriophora para el control de insectos del suelo, especialmente contra Phyllophaga spp., Cosmopolitus sordidus, larvas de lepidóptera, picudo del camote (Cylas formicarius) y termitas en el suelo. Posiblemente, el mayor aporte es en la enseñanza de las técnicas y tecnologías del control biológico de plagas que se han distribuido por América Latina a través de los graduados de Zamorano que estudiaron y fueron entrenados en Zamorano.

Palabras clave: Bioplaguicidas, control biológico clásico, enemigos naturales, entomopatógenos, parasitoides.

\section{Zamorano and its Contributions to Sustainable Agriculture through Biological Control of Pests}

\begin{abstract}
The Center for Biological Control in Central America (CCBCA) was created in 1989 by the Panamerican School of Agriculture with funds from the United States Agency for International Development (USAID) in Honduras. The CCBCA focused on classical biological control and the augmentation and conservation of natural enemies. In 2000, the name was changed to Biological Control Laboratory and the focus was placed on learning by doing for students at the Panamerican School of Agriculture and on the production and commercialization of natural enemies of agricultural pests. The only parasitoid known to attack the bromeliad weevil (Metamasius quadrilineatus) was discovered through research by the CCBCA; the species was new to science and named Lixadmontia franki, and it was released in Florida in 2007 for biological control of the Mexican bromeliad weevil (Metamasius callizona). The parasitic organisms of the pests Plutella xylostella, Mocis latipes, Spodoptera frugiperda, Leptophobia aripa, and Liriomyza spp. were inventoried. Four species of exotic parasitic wasps, one baculovirus, one fungus, and three weevils were introduced into Honduras for classical biological control: Cotesia plutellae (Asian parasitoid of P. xylostella); Diadromus collaris (pupal parasitoid of P. xylostella);

\footnotetext{
${ }^{1}$ Ph.D., University of Florida, Fort Pierce, Florida, Estados Unidos, correo electrónico rdcave@ ufl.edu

${ }^{2}$ M.Sc., Escuela Agrícola Panamericana, Zamorano, Honduras, correo electrónico rtrabanino@zamorano.edu

${ }^{3}$ Ph.D., Escuela Agrícola Panamericana, Zamorano, Honduras, correo electrónico apitty@zamorano.edu
} 
Cave et al.: Zamorano y sus Contribuciones a la Agricultura Sostenible a Través del Control Biológico de Plagas

Telenomus remus (egg parasitoid of 30 species of Lepidoptera); a species of Eretmocerus (originally from India, for control of Bemisia tabaci); the weevils Neochetina bruchi and Neochetina eichhorniae and the fungus Cercospora piaropi (for control of the weed water hyacinth (Eichhornia crassipes)); and the weevil Neohydronomous affinis (biological control agent of the weed water lettuce (Pistia stratiotes). In 2000, Zamorano changed its strategy of using biological control and began to venture into the commercial production of microorganisms for pest control, due to demand that was not being fulfilled. Biological control agents that have been produced are the antagonistic fungus Trichoderma harzianum (Trichozam ${ }^{\mathrm{TM}}$ ) for combatting fungi in the soil, Beauveria bassiana $\left(\right.$ Bazam $\left.^{\mathrm{TM}}\right)$ for controlling caterpillars and beetle larvae, Lecanicillium lecanii $\left(\right.$ Verzam $\left.^{\mathrm{TM}}\right)$ for the control of aphids and whiteflies, Metarhizium anisopliae (Metazam ${ }^{\mathrm{TM}}$ ) for control of spittlebugs (Aeneolamia spp.) and beetle larvae in sugarcane, and Purpureocillium lilacinum (Pazam ${ }^{\mathrm{TM}}$ ) for nematode control. Moreover, the predatory bug Orius insidiosus for control of thrips, aphids, and whiteflies, the predatory mite Neoseiulus longispinosus for control of spider mites (Tetranychus spp.), and the entomophagous nematode Heterorhabditis bacteriophora for control of soil insects, especially Phyllophaga spp., Cosmopolitus sordidus, caterpillars, sweetpotato weevil (Cylas formicarius), and soil termites, have been reproduced and marketed. Possibly, the greatest contribution is in the teaching of the techniques and technologies of biological pest control throughout Latina America by means of the Zamorano graduates that studied and trained in Zamorano.

Key words: Biopesticides, classical biological control, natural enemies, entomopathogens, parasitoids.

\section{Introducción}

El Centro para el Control Biológico en Centro América (CCBCA) fue creado en 1989 como parte del programa de Manejo Integrado de Plagas en Honduras (MIPH) del Departamento de Protección Vegetal (DPV) de la Escuela Agrícola Panamericana, Honduras. El programa MIPH fue financiado por la United States Agency for International Development (USAID) en Honduras. Se creó para promover el desarrollo de la enseñanza, la investigación y la implementación del control biológico en la región. El propósito fue satisfacer la necesidad de infraestructura e información técnica para brindar alternativas biológicas para el manejo de plagas en Centro América. Para lograr este propósito, el CCBCA ha colaborado con muchas instituciones internacionales. Estas incluyeron el International Institute of Biological Control (ahora CAB International), University of Florida, Purdue University, Iowa State University, Cornell University, Universidad de Costa Rica, Universidad Nacional Autónoma de Nicaragua, Centro Agronómico de Investigación y Enseñanza (CATIE) y el International Institute of Tropical Agriculture. El trabajo del CCBCA, con estas organizaciones, ha contribuido a mantener o incrementar los rendimientos de los cultivos y reducir los problemas agronómicos, económicos, ambientales y de salud pública asociados con el uso indiscriminado de plaguicidas para el control de plagas.

El CCBCA fue usado por profesores y alumnos de Zamorano, y estudiantes graduados de universidades latinoamericanas, norteamericanas y europeas para investigaciones en control biológico. Se ha utilizado el laboratorio para realizar ejercicios para cursos en Zamorano y entrenar participantes en cursos cortos. El CCBCA también se usó como base de investigación para científicos extranjeros que buscaban organismos nativos con potencial de ser agentes de control biológico en otras regiones.

El CCBCA ha trabajado en las tres estrategias que tiene el control biológico aplicado para combatir las plagas.

1. El control biológico clásico, el cual abarca el descubrimiento, importación y establecimiento de enemigos naturales exóticos, con el fin de regular poblaciones de plagas introducidas o nativas en un país o región determinada.

2. El aumento de enemigos naturales por la cría masiva y liberación en el campo o invernadero para aumentar las poblaciones de enemigos naturales.

3. La conservación de enemigos naturales, definida como el uso de prácticas agronómicas y hortícolas que hace el ambiente del agroecosistema más favorable a la protección y mantenimiento de poblaciones de enemigos naturales. 


\section{Inventarios de los Enemigos Naturales de Plagas}

Los inventarios de los enemigos naturales de plagas son importantes para dos propósitos. Un propósito es conocer los organismos que contribuyen a la mortalidad de la plaga, con el fin de llevar a cabo estudios ecológicos detallados que determinan cuales factores desempeñan un papel significativo en la regulación de la población de la plaga. Esta información es importante en el desarrollo de tecnologías para la conservación de enemigos naturales y para la selección de enemigos naturales para proyectos de aumento. Segundo propósito es indicar cuales enemigos naturales no están presentes y, por lo tanto, deben ser considerados para importación en proyectos de control biológico clásico.

Cuatro especies de plagas lepidópteras tuvieron sus organismos parasíticos bien inventariados. Plutella xylostella (L.) (Lepidoptera: Plutellidae), la palomilla de dorso de diamante, es huésped de tres especies de parasitoides primarios y dos especies de parasitoides facultativos (Cordero y Cave 1992). Treinta y una especies parasitan los cuatro estados de Mocis latipes (Guenée) (Lepidoptera: Noctuidae), el gusano de las gramíneas, en el sur-centro de Honduras (Cave 1992). Cave (1993) listó 43 especies de parasitoides en Centroamérica que atacan a las larvas y pupas de Spodoptera frugiperda (Smith) (Lepidoptera: Noctuidae), el gusano cogollero, y creó una clave para identificar las 26 especies conocidas de Honduras. Cave y Cordero (1999) reportaron 12 especies de moscas y avispas parasíticas que atacan a las larvas y pupas de Leptophobia aripa Boisduval (Lepidoptera: Pieridae), la mariposa mejicana de la col.

Veinte y cinco especies de Hymenoptera atacan a tres especies de Liriomyza (Diptera: Agromyzidae) que son minadores de las hojas de varios cultivos y malezas en el sur de Honduras (Acosta y Cave 1994). Chrysonotomyia diastatae (Howard) (Eulophidae), Opius dissitus Muesebeck (Braconidae) y Ganaspidium utilis Beardsley (Figitidae) fueron los parasitoides más abundantes. Acosta y Cave (1994) también proporcionaron una clave para facilitar la identificación de los parasitoides de Liriomyza en Centroamérica.

Cave y Márquez (1994) presentaron un inventario de las 25 especies de avispas parasíticas que atacan a las especies de Diaspididae, Coccidae y Aleyrodidae en cítricos en Honduras, y discutieron los candidatos potenciales para importación en programas de control biológico clásico. Cave (1994) listó 10 especies de himenópteros parasitando la mosca blanca de la batata, Bemisia tabaci (Gennadius) (Hemiptera: Aleyrodidae), en Centroamérica. Bográn et al. (1998) reportaron que el porcentaje de parasitismo varió entre 21 y $32 \%$ en la siembra de primera y entre 10 y $37 \%$ en la de postrera; los parasitoides recolectados más frecuentemente fueron Encarsia pergandiella Howard y Encarsia nigricephala Dozier (Hymenoptera: Aphelinidae).

Cave (1997) reportó el hallazgo del único parasitoide conocido que ataca al picudo de las bromelias, Metamasius quadrilineatus Champion (Coleoptera: Curculionidae). Luego, Wood y Cave (2006) describieron y nombraron el parasitoide Lixadmontia franki Wood y Cave (Diptera: Tachinidae). La mosca parasítica fue liberada en Florida en 2007-2010 para establecer poblaciones en ese estado para el control biológico del picudo mejicano de las bromelias, Metamasius callizona (Chevrolat) (Cave 2008)

Mucha de la información de estos inventarios formó la base del Manual para el Reconocimiento de Parasitoides de Plagas Agrícolas en América Central por Cave (1995a). Este libro ofrece información sobre la crianza de parasitoides, métodos de montaje, morfología y también una clave para las familias de Hymenoptera parasítica y un listado de trabajos taxonómicos para la identificación de parasitoides de plagas agrícolas en Centroamérica. El manual facilita el reconocimiento de 112 especies de parasitoides primarios y tres especies de hiperparasitoides de los órdenes Diptera e Hymenoptera, dando un diagnóstico, hospederos conocidos, distribución, notas biológicas y uno o más dibujos por especie. Además, el manual provee un listado extenso de las publicaciones sobre la taxonomía y biología de las especies presentadas.

\section{Introducción de Enemigos Naturales para el Control de Plagas}

Cuatro especies de avispas parasíticas exóticas y un baculovirus fueron introducidas a Honduras para control biológico clásico. Cotesia plutellae (Kurdjumov) (Hymenoptera: Braconidae) es un para- 
sitoide asiático de $P$. xylostella y 10,445 individuos de esta avispa fueron liberado en más de tres sitios en Honduras entre diciembre de 1988 y noviembre de 1989 (Cherry 1991). Aunque se recuperaron 33 individuos de la generación $\mathrm{F}_{1}$, no hubo evidencia que las poblaciones de $C$. plutellae se establecieron. Menos de 200 adultos del parasitoide pupal Diadromus collaris (Gravenhorst) (Hymenoptera: Ichneumonidae) fueron liberados en 1990, pero no hubo evidencia de su establecimiento en el campo.

Telenomus remus Nixon (Hymenoptera: Platygastridae) es un parasitoide ovífago de origen asiático que ataca a los huevos de 30 especies de lepidópteros (Cave 2000). En Honduras, T. remus fue liberado en campos de maíz y sorgo de 1991 a 1994 (Cave y Acosta 1999). Las tasas de parasitismo variaron entre 20 y $92 \%$, con mayores niveles de parasitismo en las parcelas con buen crecimiento del cultivo y la presencia de malezas en floración (65$92 \%$ ) que en las parcelas con mal crecimiento del cultivo y no malezas (20-60\%).

De agosto a diciembre de 1994, se liberaron en Comayagua 2,787 adultos de una especie de Eretmocerus (Hymenoptera: Aphelinidae) originaria de Thirumala, India (Gómez Cabrera 1995). El propósito de estas liberaciones fue establecer poblaciones viables en el campo para incrementar el control biológico de $B$. tabaci. Los parasitoides fueron enviados del Laboratorio de Control Biológico en Mission, Texas, Estados Unidos. En muestras tomadas de septiembre 1994 hasta febrero de 1995 en 13 plantas hospederas, el parasitismo varió de 0 a $3 \%$ en B. tabaci, y de 0 a $52 \%$ en Trialeurodes spp. en ocho plantas hospederas (Gómez Cabrera 1995); estos niveles de parasitismo fueron muy bajo en comparación con el parasitismo por E. pergandiella. Cave (1994) argumentó que el control biológico de $B$. tabaci sería más efectivo dentro de un programa integrado con métodos de control no químicos.

La maleza lirio de agua, jacinto de agua, jacinto acuático, lechuguin o buchón (Eichhornia crassipes (Martius) Solms-Laubach) es la maleza acuática más problemática del mundo. Es nativa del Amazona, pero se ha distribuido por todas las áreas tropicales y subtropicales (Holm et al. 1977). No se conoce como llegó a Honduras, pero ha estado establecida desde inicios del siglo XX. En Zamorano ha estado establecida posiblemente desde inicios de la institución en 1942.

En Zamorano, la maleza cubría por lo menos la mitad de la superficie del lago de Monte Redondo en 1990 (Figura 1). Todos los sábados, los estudiantes del módulo de acuacultura pasaban la mañana sacando a mano las plantas; en ocasiones las tardes también las dedicaban a esta actividad. Los estudiantes de acuacultura empezaron a limpiarlo a mano en 1984, pero el problema nunca terminaba, la maleza crecía a igual o mayor velocidad que la que se podía remover.

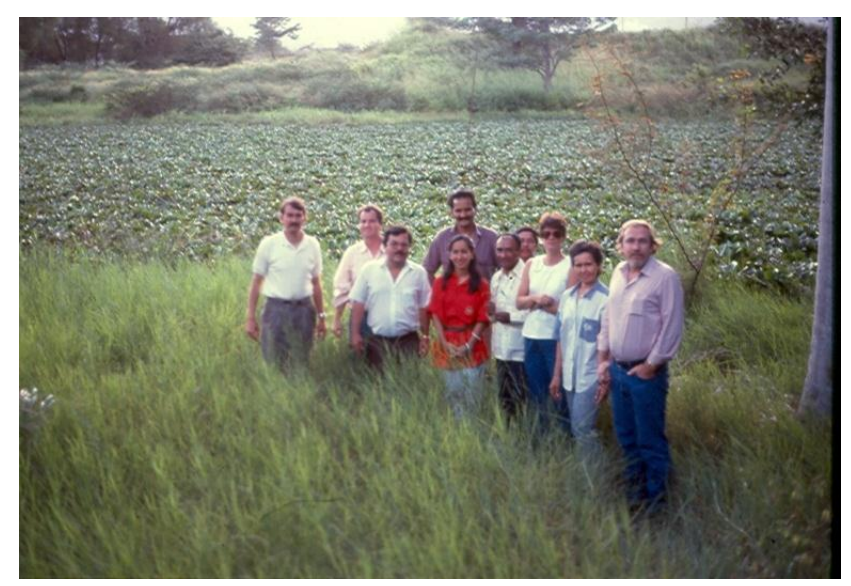

Figura 1. Participantes en la Primera Reunión del Panel de Especialistas Centroamericanos en Control Biológico, agosto de 1990, izquiera a derecha: Tomas Zoebisch, Ronald D. Cave, Manuel Carballo, Francisco Badilla, Ligia Lacayo, Ronald Estrada, Tito Antón, Sally Gladstone, Maritza Vargas y Philip Shannon. Al fondo el lago de Monte Redondo cubierto por el lirio acuático (vista Sur-Oeste).

Los picudos Neochetina bruchi Hustache y Neochetina eichhorniae Warner (Coleoptera: Curculionidae) fueron introducidos a Honduras el 12 de febrero de 1990. Se introdujeron 24 adultos de $N$. bruchi y 123 de N. eichhorniae (Pitty 2004). La introducción siguió todos los protocolos establecidos por el gobierno de Honduras para la introducción de organismos vivos. Los insectos llegaron de Florida con la cooperación de Fred Bennett de la Universidad de Florida. La introducción la hizo el CCBCA, dirigido por Ronald D. Cave. Abelino Pitty (especialista en manejo de malezas) estuvo a cargo del seguimiento al establecimiento de los picudos. 
La liberación se hizo en el lago de Monte Redondo en Zamorano. El 84\% de los insectos liberados eran $N$. eichhorniae y $16 \% \quad N$. bruchi. Debido a que $N$. bruchi tiene un ciclo de vida más corto y pone más huevos, la proporción de $N$. bruchi había aumentado en agosto de 1991 (19 meses después) a 37\%. Neochetina bruchi pone 2.6 huevos por día y el ciclo de vida es de 73 días, $N$. eichhorniae pone 2.1 huevos por día y el ciclo de vida es de 94 días (Njoka et al. 2006). Los picudos limpiaron el lago de Monte Redondo (Figura 2 y 3) de manera permanente y no hay que hacer control mecánico, ya que se alimentan de las hojas y reducen la fotosíntesis al comer las hojas e introducir hongos y bacterias que dañan las hojas (Venter 2013); y las larvas perforan los estolones que luego se pudres y se hunden.

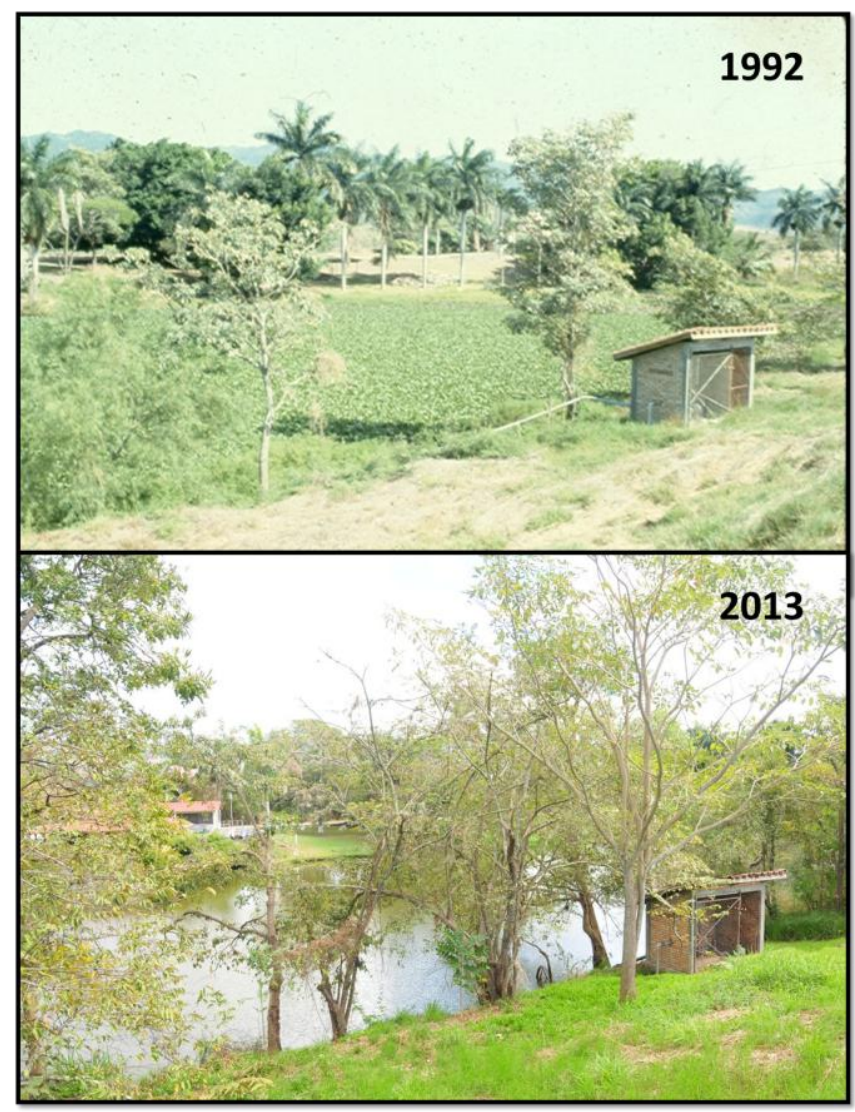

Figura 2. Arriba, el lago de Monte Redondo bastante infestado con el lirio acuático en 1991, abajo el mismo lago en el 2013 ya libre del lirio acuático (vista al Sur).

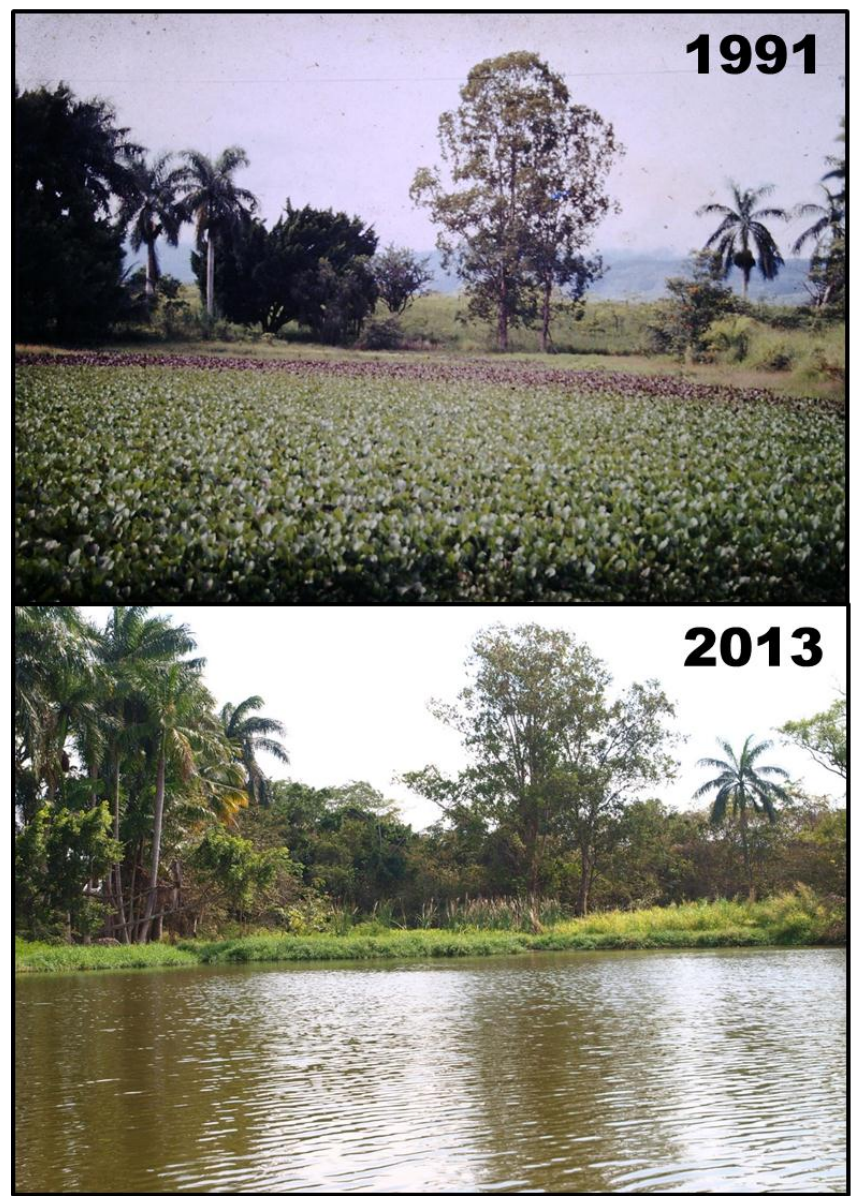

Figura 3. Arriba, el lago de Monte Redondo infestadocon el lirio acuático en 1991, abajo el mismo lago libre del lirio acuático en el 2013 (vista al Sur).

Los picudos fueron llevados el 7 de febrero de 1992 al Lago Yojoa por R. D. Cave, Isaac Dejud y A. Pitty (Figura 4), con la colaboración de la Empresa Nacional de Energía Eléctrica (ENEE). La liberación fue cerca del Hotel Lago Azul y del Hotel Los Remos. Los picudos se establecieron rápidamente.

El 28 de abril de 1994 se liberaron en la represa Los Laureles, Tegucigalpa, por A. Pitty, Helen Mero e Ian Zelaya; hubo otra en Ceiba, Honduras, en colaboración con el Centro Universitario Regional del Litoral Atlántico (CURLA). De Zamorano, los picudos han sido llevados a El Salvador por Leopoldo Serrano, profesor en la Universidad de El Salvador. A Cuba fue llevada la especie $N$. bruchi por David Greathead a 
través de la FAO; N. eichhorniae ya estaba establecida en Cuba. No se conoce el resultado de estas introducciones.

Los picudos están establecidos en el Lago Yojoa. En el 2002 se observaron cerca del Hotel Lago Azul las marcas que dejan los adultos en las hojas y peciolos, pero no se encontraron los adulto. El 2 de septiembre de 2006 fueron encontrados por A. Pitty y M. Owen (Iowa State University) cerca del Hotel Lago Azul. El 17 de enero de 2011 se encontraron 13 picudos de $N$. eichhorniae y 4 de $N$. bruchi por A. Pitty, George Pilz, Welsy Vásquez (Diputada por el departamento de Cortes) y Emilio Cruz (Diputado por el departamento de Comayagua).

El hongo Cercospora piaropi (Tharp) (=Cercospora rodmanii (Conway)) fue introducido y liberado en Honduras por Zamorano en febrero de 1991, por Luis del Río y A. Pitty. El hongo no se recuperó después de la liberación, se supone que no se estableció porque en febrero, marzo y abril hay poca lluvia y humedad relativa baja, y baja su reproducción.

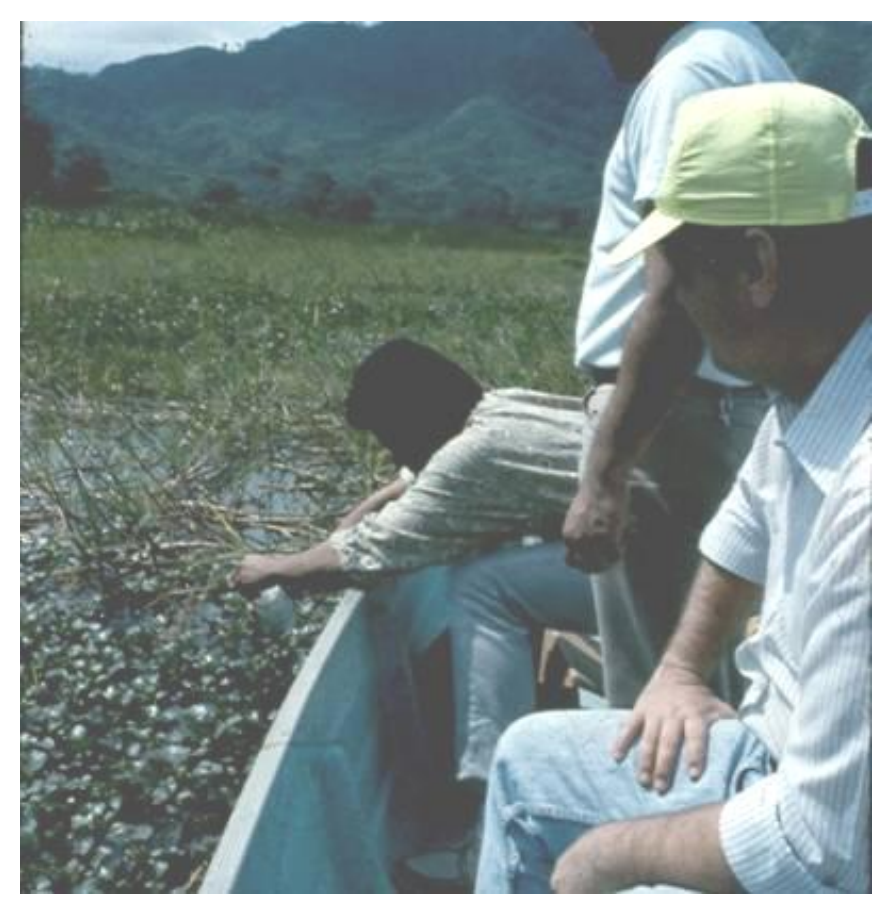

Figura 4. Isaac Dejud, estudiante de Zamorano, liberando los picudos el 7 de febrero de 1992 en el Lago Yojoa, Honduras, al frente el Dr. Ronald D. Cave.
El picudo Neohydronomous affinis Hustache, nativo de Suramérica, fue introducido en 1995 y liberado en la laguna Ticamaya, Departamento de Cortes, Honduras, por Abelino Pitty y Gisela Godoy; vinieron de Florida, ahí habían sido introducido en 1987. El objetivo fue para controlar la maleza acuática lechuga de agua (Pistia stratiotes L.) que está distribuida alrededor del mundo en lugares tropicales y subtropicales (Holm et al. 1977). En Honduras está en el Lago Yojoa, la laguna Ticamaya $y$ en Comayagua. No se conoce si se establecieron.

\section{Otras Investigaciones}

La relación entre la dosis aplicada del virus de poliedrosis nuclear (VPN) Galleria mellonela (L.) y el tamaño de la larva de $P$. xylostella fue estudiado en el laboratorio por Espinoza et al. (1994). La mortalidad de $95 \%$ de larvas ocurrió a las 120 horas de inoculación y la mortalidad fue semejante entre larvas de segundo y tercer estadio. En el campo, la mortalidad de larvas se redujo más de $40 \%$ cuando las plantas asperjadas con VPN G. mellonela eran expuestas a la luz directa del sol al menos 4 horas, y la tasa de empupación se redujo hasta $79 \%$ en plantas sin exponer al sol comparada con el testigo de exposición completa al sol (Espinoza y Cherry 1994).

Escribano et al. (1999) compararon biológica, genética y estructuralmente cuatro cepas del VPN $S$. frugiperda aislados de larvas de Estados Unidos, Nicaragua y Argentina para seleccionar el candidato para experimentos de campo en Honduras y México. Las cepas no fueron iguales genéticamente y la de Nicaragua y una de Estados Unidos causaron la mayor infectividad. Pruebas de campo en Honduras y México produjeron resultados semejantes en la mortalidad de S. frugiperda después de aplicaciones al maíz con VPN $S$. frugiperda o clorpirifos (Williams et al. 1999). La dosis más alta del virus causó $40 \%$ de mortalidad de larvas. El parasitismo por avispas y moscas contribuyó otro $40 \%$ de mortalidad en las parcelas aplicadas con el virus; la reducción de los enemigos naturales por clorpirifos, aparentemente, causó un resurgimiento de $S$. frugiperda en parcelas aplicada con clorpirifos. Añadir azúcar a la formulación viral para estimular la alimentación no aumentó la probabilidad de infección. Santos Erazo (1998) 
demostró que el parasitismo por T. remus en el campo no es reducido por las aplicaciones del baculovirus $S$. frugiperda. Williams et al. (1999) también notaron que el uso de VPN $S$. frugiperda fue más costoso que el control químico debido a los costos de producción y aplicación del virus, pero los costos podrían ser menores con mano de obra más eficiente y reducción de los costos de materiales en la dieta artificial.

Los insecticidas siempre serán importantes en el manejo integrado de plagas. Por lo tanto, el efecto de los insecticidas en los enemigos naturales de las plagas es un campo de estudio valioso. Cherry (1991) determinó que profenofos + cipermetrina causó la mayor mortalidad de C. plutellae y Diadegma insulare (Cresson), dos parasitoides de la palomilla de dorso de diamante, en comparación con el insecticida bacterial Dipel WP (Bacillus thuringiensis Berliner) y clorfluazuron, un regulador de crecimiento.

Los controles culturales a veces aumentan las poblaciones y actividades de depredadores y parasitoides. Morjan Erazo (1993) encontró más diversidad de depredadores nocturnos en maíz sembrado en el sistema de labranza cero (LCE) que en el sistema de labranza convencional (LCO). González y Cave (1997a) reportaron que las arañas foliares diurnas eran 1.2 veces más en frijol en LCE que en LCO. Del mismo modo, las tasas de parasitismo de los huevos de Empoasca kraemeri Ross y Moore, el lorito verde, por el parasitoide ovífago Anagrus gonzalezae Triapitsyn fueron 2-3 veces mayores en frijol en postrera con LCE en comparación con el cultivo bajo LCO (González y Cave 1997b).

Cañas Castro (1996) comparó la abundancia de enemigos naturales y la dinámica de las poblaciones del gusano cogollero en maíz en parcelas aplicadas con azúcar o con agua solamente. En general, encontró más enemigos naturales en el maíz aplicado con azúcar que sin azúcar. La hormiga brava Solenopsis geminata (F.) y la mosca parasítica Lespesia archippivora (Riley) fueron más abundantes en el maíz con azúcar. El daño a las hojas por el gusano cogollero fue reducido por $35 \%$ en el maíz tratado con azúcar en comparación al maíz sin azúcar. Las tasa de infestación de plantas fue $19 \%$ más bajo en las parcelas aplicadas con azúcar. Los resultados indican que la mayor cantidad de enemigos naturales atraídos al maíz aplicado con azúcar reduce significativamente las poblaciones y el daño por el gusano cogollero.

Cordero et al. (2000) mostraron que el manejo de malezas es una práctica clave en el control de plagas lepidópteras en sorgo y maíz. Cultivos bajo manejo de malezas tradicional (eliminación al momento de siembra) tuvieron mayor densidad de larvas por planta comparados con cultivos con manejo retrasado (eliminación de malezas tres semanas después de la siembra). Sin embargo, las larvas en cada sistema de manejo de malezas sufrieron iguales niveles de parasitismo (11-16\%), lo cual indica que los 12 especies de parasitoides no fueron afectado por el sistema de manejo de malezas.

\section{Capacitaciones}

El CCBCA proveyó a los estudiantes del programa de Ingeniería Agronómica de Zamorano la oportunidad de conducir su investigación de tesis. Adicionalmente, varios estudiantes graduados realizaron sus investigaciones en colaboración con el CCBCA. Los temas (Cuadro 1) abarcaron una diversidad de estudios que contribuyeron significativamente a los conocimientos del control biológico en Centro América.

En 1993, hubo un taller importante sobre la enseñanza del control biológico en América Latina para fortalecer la capacidad de los instructores universitarios del tema. Diez participantes de seis países de Centro y Sur América participaron. Un producto valioso del taller fue el libro Manual para la Enseñanza del Control Biológico en América Latina editado por R.D. Cave (1995b).

Los éxitos del uso del hongo entomopatógeno Metarrhizium anisopliae (Metchnikoff) Sorokin (Hypocreales: Clavicipitaceae) en Costa Rica y Brasil para controlar salivazos (Aeneolamia spp. y Prosapia spp. [Hemiptera: Cercopidae]) en caña de azúcar, impulsaron la presentación del Taller sobre la Tecnologías de Producción Masiva y Aplicación de Metarrhizium anisopliae en 1990 en Zamorano. Subsecuentemente, el hongo fue producido en el CCBCA y aplicado en los cañaverales en Honduras por unos pocos años. Lastimosamente, las aplicaciones en el campo no rindieron los resultados esperados y se descontinuó la producción de $M$. anisopliae, hasta su reactivación en el 2004. 
Cave et al.: Zamorano y sus Contribuciones a la Agricultura Sostenible a Través del Control Biológico de Plagas

Cuadro 1. Títulos de tesis y disertaciones realizadas por estudiantes en cooperación con el CCBCA.

Acosta, N. 1992. Inventario y evaluación de enemigos naturales de Liriomyza spp. (Diptera: Agromyzidae) en la región sur de Honduras. Tesis Ing. Agr., Escuela Agrícola Panamericana, El Zamorano, Honduras.

Álvarez del Hierro, D.R. 1997. Biología reproductive y método de crianza en el laboratorio de Admontia sp. (Diptera: Tachinidae), parasitoide de Metamasius quadrilineatus Champion (Coleoptera: Curculionidae). Tesis Ing. Agr., Escuela Agrícola Panamericana, El Zamorano, Honduras.

Bográn, C. E. 1996. Biological control of the whitefly Bemisia tabaci (Gennadius) on common bean (Phaseolus vulgaris L.) in Honduras. Tesis MSc, Iowa State University, Ames, IA.

Bonilla Vásquez, K.B. 2000. Control biológico de Helicoverpa zea con Trichogramma pretiosum y Bacillus thuringiensis en tomate. Tesis Ing. Agr., Escuela Agrícola Panamericana, El Zamorano, Honduras.

Cañas Castro, L.A. 1996. Response of natural enemies to sugar applications in maize and their effect on fall armyworm Spodoptera frugiperda (J. E. Smith) populations in Honduras. Tesis M.Sc, Purdue University, West Lafayette, IN.

Cañas Castro, L.A. 2000. Population ecology of Leptinotarsa undecimlineata Stål (Coleoptera: Chrysomelidae) and their effects on Solanum lanceolatum Cav. in Honduras. Disertación PhD, Purdue University, West Lafayette, IN.

Carrillo Vilchez, M.A. 1997. Metodología de cría semiartesanal de Chrysoperla externa (Hagen) (Neuroptera: Chrysopidae) y su liberación en repollo para el control de Plutella xylostella (L.). Tesis Ing. Agr., Escuela Agrícola Panamericana, El Zamorano, Honduras.

Castelo Ipiales, M.Y. 1999. Biología reproductive y análisis electroforético de Diadegma insulare y Diadegma semiclausum (Hymenoptera: Ichneumonidae). Tesis Ing. Agr., Escuela Agrícola Panamericana, El Zamorano, Honduras.

Cherry, A. 1991. Aspects of the interaction between biological and chemical control methods for Plutella xylostella (L.) (Lepidoptera: Yponomeutidae). Disertación Ph.D. University of London, Imperial, College, UK.

Cordero, R.J. 1989. Parasitoides de Plutella xylostella L. (Lepidoptera: Plutellidae) en cultivo de repollo en Honduras. Tesis Ing. Agr., Escuela Agrícola Panamericana, El Zamorano, Honduras.

Cordero Alonso, R.J. 1999. Relationships of weed management and parasitoids with lepidopterous pests in intercropped sorghum and maize in southern Honduras. Tesis MSc, Mississippi State University, Mississippi State, MS.

Díaz Galárraga, R.R. 1999. Control biológico del gusano elotero (Helicoverpa zea (Boddie)) en maíz dulce producido en Zamorano. Tesis Ing. Agr., Escuela Agrícola Panamericana, El Zamorano, Honduras.

Espinoza, A. 1994. Utilización de baculovirus para el control de la palomilla dorso de diamante, Plutella xylostella (L.) (Lepidoptera: Plutellidae). Tesis Ing. Agr., Escuela Agrícola Panamericana, El Zamorano, Honduras.

Gavilánez, J. 1994. Diagnóstico de campo, pruebas experimentales y estudio de prefactibilidad para la cría masiva de Chrisopidae [sic] Neuroptera en Honduras. Tesis Ing. Agr., Escuela Agrícola Panamericana, El Zamorano, Honduras.

Gómez Cabrera, L.E. 1995. Control biológico clásico de Bemisia tabaci (Gennadius) en Honduras. Tesis Ing. Agr., Escuela Agrícola Panamericana, El Zamorano, Honduras.

González, A. 1993. Elaboración y evaluación de cursos de control biológico para agricultores y extensionistas. Tesis Ing. Agr., Escuela Agrícola Panamericana, El Zamorano, Honduras.

González, A. 1994. Control biológico de Empoasca kraemeri (Homoptera: Cicadellidae) en dos sistemas de labranza. Tesis Ing. Agr., Escuela Agrícola Panamericana, El Zamorano, Honduras.

González, E. 1993. Polybia spp. como depredadores en el Valle del Yeguare, Honduras. Tesis Ing. Agr., Escuela Agrícola Panamericana, El Zamorano, Honduras. 
Cuadro 1. Continuación.

González, M. 1994. Dinámica poblacional y parasitismo de la mosca doméstica, Musca domestica, y la mosca del establo, Stomoxys calcitrans. Tesis Ing. Agr., Escuela Agrícola Panamericana, El Zamorano, Honduras.

González Hernández, J.M. 1999. Reciclaje de VPN Spodoptera frugiperda en el campo para el control del gusano cogollero S. frugiperda (J. E. Smith) en el cultivo de maíz. Tesis Ing. Agr., Escuela Agrícola Panamericana, El Zamorano, Honduras.

Márquez, J.G. 1990. Complejo de parasitoides de Coccidae, Diaspididae y Aleyrodidae en el cutivo de cítricos en Honduras. Tesis Ing. Agr., Escuela Agrícola Panamericana, El Zamorano, Honduras.

Méndez Castellanos, M.A. 1997. Efectividad de hongos y nematodos entomopatógenos para el control de las gallinas ciegas, Phyllophaga spp., en Miraflor, Estelí, Nicaragua. Tesis Ing. Agr., Escuela Agrícola Panamericana, El Zamorano, Honduras.

Molina Cox, M.A. 1999. Evaluación de insecticidas botánicos, biológicos y sintéticos sobre Trichogramma pretiosum, Diadegma insulare, Chrysoperla carnea e Hippodamia convergens. Tesis Ing. Agr., Escuela Agrícola Panamericana, El Zamorano, Honduras.

Morjan Erazo, W.E. 1993. Depredadores nocturnos de plagas de maíz y de frijol en dos sistemas de labranza. Tesis Ing. Agr., Escuela Agrícola Panamericana, El Zamorano, Honduras.

Novillo Rameix, P. 1991. Prostephanus truncatus (Horn (Coleoptera: Bostrichidae) en el ambiente de almacenimiento tradicional de maíz en el Valle de El Zamorano Honduras, C. A. Tesis Ing. Agr., Escuela Agrícola Panamericana, El Zamorano, Honduras.

Pérez, A. 1993. Capacitación de pequeños productores de crucíferas de San Juan del Rancho sobre control biológico de Plutella xylostella L. Tesis Ing. Agr., Escuela Agrícola Panamericana, El Zamorano, Honduras.

Pérez, C. 1995. Managing resistance to Bacillus thuringiensis in Plutella xylostella (Lepidoptera: Plutellidae) in the field. Disertación PhD, Cornell University, Ithaca, NY.

Román Suárez, D.X. 1998. Bioensayos de campo y análisis económico de la producción del virus de la poliedrosis nuclear Spodoptera frugiperda. Tesis Ing. Agr., Escuela Agrícola Panamericana, El Zamorano, Honduras.

Santos Erazo, F.E. 1998. Uso combinado de VPN Spodoptera frugiperda, Telenomus remus, y aplicaciones de azúcar para el control biológico del cogollero, Spodoptera frugiperda, en maíz. Tesis Ing. Agr., Escuela Agrícola Panamericana, El Zamorano, Honduras.

Vélez Izaguirre, J.J. 1993. Relación entre la etapa fenológica y la variedad de frijol con el nivel de parasitismo de Bemisia tabaci Gennadius. Tesis Ing. Agr., Escuela Agrícola Panamericana, El Zamorano, Honduras.

\section{Un Enfoque Diferente, el Control Biológico Comercial}

En el 2000, el Ing. Rogelio Trabanino se hizo cargo del CCBCA y cambió el enfoque y el nombre a Laboratorio de Control Biológico. Al inicio, el enfoque fue seguir la enseñanza de las bases del control biológico siguiendo lo que R.D. Cave había dejado como legado. El inicio fue muy difícil porque ya no existía el personal que había trabajado en el CCBCA y se tuvo que capacitar al nuevo personal, y con poco presupuesto seguir por lo menos con la enseñanza de los principios del control biológico.
Siguiendo la filosofía del aprender-haciendo de Zamorano, el Laboratorio de Control Biológico produjo $T$. remus y el VPN $S$. frugiperda que sirvieron para las prácticas, la producción se usaba para suplir algunos clientes que todavía usan estos organismos. Deespués, el laboratorio empezó a recibir solicitudes de agricultores requiriendo ayuda para controlar plagas en la producción de hortalizas, lo que hizo orientar la producción para suplir la demanda.

En giras por Cuba, Bolivia y Estados Unidos, se conoció la parte comercial y reproductiva de estos organismos. Se estableció relación con el proyecto FINTRAC que trataba de incrementar los ingresos de 
los productores enseñándoles a producir hortalizas. El proyecto fue financiado por USAID, así que no podían usar unos agroquímicos muy populares, pero toxicológicamente muy dañinos para el hombre y el ambiente. FINTRAC solicitó que produjeran plaguicidas biológicos para reducir la mortalidad en el campo de las plántulas de chile jalapeño, que era de 40-50\%. Esta alianza permitió dedicar esfuerzos a la enseñanza, a la investigación aplicada, a la producción de un organismo para los productores de hortalizas y a la extensión en evaluaciones en el campo.

Se decidió reproducir el hongo antagonista Trichoderma harzianum Rifai para combatir hongos del suelo. Se probó la eficacia, rapidez de crecimiento y rendimiento para seleccionar las cepas adecuadas. Estas evaluaciones fueron con el Dr. Phil Arneson (Cornell Univ.y y profesor adjunto de Zamorano) y un grupo talentoso de estudiantes de Zamorano. Después de las pruebas de efectividad en el laboratorio, se evaluaron en el campo con cultivos hortícolas; los resultados demostraron la efectividad del producto. Tres años después (2003), se registró en Honduras el primer biopesticida de Zamorano con el nombre comercial de Trichozam $^{\mathrm{TM}}$. Los datos para el registro inicial y los siguientes fueron obtenidos de las tesis de estudiantes (Méndez Martinez 2003; Medina Teran 2003; Michel Marco 2003 y Raudes Reyes 2006).

Seis meses después de usar Trichozam ${ }^{\mathrm{TM}}$ en chile jalapeño, la mortalidad por damping off se redujeron a $5 \%$, y el producto fue adoptado por la mayoría de los exportadores. En el 2003, se estimó que unas 300 ha de chile jalapeño, 1,300 de tomate y unas 1,800 de chile dulce se aplicaban con Trichozam ${ }^{\mathrm{TM}}$.

En el 2004, se registró el bioplaguicida Bazam ${ }^{\mathrm{TM}}$ (Beauveria bassiana (Bals.-Criv.) Vuill), y Verzam ${ }^{\mathrm{TM}}$ (Lecanicillium lecanii (Zimm.) Zare y W. Gams). Bazam $^{\mathrm{TM}}$ controla lepidópteros, coleópteros adultos, larvas del suelo y la broca del café. Los estudios para su desarrollo y registros fueron las tesis de Romero Villagra (2004), Rivera Jerez (2004), Toapanta Valencia (2005), Espinoza Silva (2005), Gómez Pineda y Montemayor Aizpurúa (2005) y Méndez Gonzales (2008). Bazam ${ }^{\mathrm{TM}}$ se usa en cebolla contra trips y garrapatas. El Verzam ${ }^{\mathrm{TM}}$ se registró para el control de áfidos y mosca blanca, pero no tuvo éxito porque el uso de fungicidas en los cultivos hortícolas es muy frecuentes y la cepa no era muy agresiva.
En el 2004, se buscaron cepas de M. anisopliae en el Sur de Honduras, y Purpureocillium lilacinum (Thom) Luangsa-ard, Hou-braken, Hywel-Jones y Samson en Zamorano; los microorganismos fueron registrados en Honduras ante la Secretaria de Agricultura y Ganadería (SAG) en 2006 con los nombres de Metazam $^{\mathrm{TM}}$ y Pazam $^{\mathrm{TM}}$, respectivamente. El Metazam ${ }^{\mathrm{TM}}$ controla el salivazo en caña de azúcar. Unas 8,000 ha se fumigan con Metazam ${ }^{\mathrm{TM}}$ en tres ingenios azucareros en Honduras, lo que ha reducido el uso de agroquímicos. Metazam ${ }^{\mathrm{TM}}$ se usa contra gallina ciega (Phyllophaga spp.) en camote, ya que no se deben aplicar insecticidas sintéticos 30 días después de la siembra. Pazam ${ }^{\mathrm{TM}}$ es un hongo que controla nematodos y se puede utilizar en cultivos hortícolas, granos básicos y frutales. Las tesis de Vaquedano Gámez (2006), Fernandez Tondelli (2006) y Juárez Figueroa (2007) ayudaron al desarrollo de Pazam ${ }^{\mathrm{TM}}$.

En el 2005, se registró Trichozam ${ }^{\mathrm{TM}}$ en Jamaica y desde entonces se hacen envíos anuales a Jamaica. Se registró Trichozam ${ }^{\mathrm{TM}}$ y Bazam $^{\mathrm{TM}}$ en el 2008 y en el 2009 Metazam $^{\mathrm{TM}}$ y Pazam ${ }^{\mathrm{TM}}$ en El Salvador. En el 2010, los cuatro productos fueron registrados en Nicaragua. Hay gestiones de registro en República Dominicana, Guatemala y Panamá. Las tesis de Morán Quintero (2007), Castillo Samudio (2007), Moran Ruiz (2007), Parada López (2007), Mantilla Compte (2007) y Rosero Asqui (2008) han servido en esta nueva estrategia de mercado.

En el 2008, se obtuvo con el Dr. Alfredo Rueda una donación de la Cuenta del Milenio para criar, liberar y usar tres enemigos naturales para reducir el uso de agroquímicos en hortalizas. Se seleccionaron la chinche depredador Orius insidiosus (Say) para el control de trips, áfidos y mosca blanca; el ácaro depredador Neoseiulus longispinosus (Evans) para el control de la arañita roja Tetranychus spp.; y el nematodo entomófa|go Heterorhabditis bacteriophora Poinar para controlar insectos del suelo, especialmente Phyllophaga spp., Cosmopolitus sordidus (Germar), larvas de lepidóptera, picudo del camote (Cylas formicarius (F.)) y termitas en el suelo.

Este proyecto convenció a los agricultores que la agricultura sostenible es la mejor estrategia para permanecer en el mercado. Se contrataron cinco graduados de Zamoranos: Miguel Cocom (trabajó en el Laboratorio de Control Biológico); Carlos Montufar 
y Alicia Joya, (desarrollaron las crías de los organismos); Jorge Chavarría y Karla Andino (trasladaron a los agricultores los conocimientos sobre el uso del control biológico en fincas). Se construyó un laboratorio para producir H. bacteriophora y $O$. insidiosus, dos cuartos fríos de almacenamiento, y seis invernaderos computarizados para criar $N$. longispinosus. Se capacitaron 800 agricultores, 150 técnicos y personas interesadas en la producción de estos organismos para hacer pública las estrategias de cría desarrolladas y que pudieran usarlas o iniciar algún negocio. Además, 600 estudiantes aprendieron tecnologías de punta y la biología, ecología y cría de otros enemigos naturales. Hubo 15 investigaciones con los alumnos y personal técnico. Los resultados reforzaron las enseñanzas en Zamorano, a los agricultores y los técnicos. Los estudios con fondos de este proyecto fueron los de Suazo López (2008), Pantoja Guaman (2009), Perrera Viamill (2009), Turcios Rivas (2009), Turcios Rivera (2009), Villaroel Basantes (2009), Ávila Sosa (2010) y Dieguez Gonzáles (2010). Ávila Sosa (2010) y Dieguez Gonzáles (2010).

Un caso exitoso dejó grandes lecciones sobre el control de plagas, se dio asistencia técnica a dos exportadores de chile de colores cuyo problema principal era la arañita roja Tetranychus urticae Koch. El único control en 140 ha, entre ambas exportadoras. era aplicar, sin éxito, acaricidas 1.5 veces por semana a un costo de US\$6,200/ha. A fin del 2009, se liberó $N$. longispinosus y se logró reducir a dos aplicaciones de acaricidas en todo el ciclo en el primer año y ninguna en el segundo año. En el 2012, no se liberó ningún depredador ya que $N$. longispinosus está establecido en los alrededores de los invernaderos, lo que impide la reproducción de la plaga y su introducción a los invernaderos. Los productores ya no gastan más en el control de la arañita roja. Otro éxito fue con el cultivo de camote en la empresa Monty Farm, una de las mayores exportadoras de Honduras. En el 2010, se reportaron daños del picudo del camote que perfora los tubérculos y reduce su calidad y comercialización. Las prácticas de control químico no son efectivas ya que el picudo está en el suelo y es muy móvil, por lo cual se evaluó el efecto de $H$. bacteriophora sobre larvas y adultos y después de un ciclo de aplicaciones, la efectividad fue $95 \%$.

El laboratorio de Control Biológico evalúa la producción de hongos que puedan aumentar la cartera de productos para la enseñanza. Ha comenzado a producir algunas bacterias para que los estudiantes aprendan y puedan producir organismos que controlan plagas, incluyendo Bacillus subtilis (Ehrenberg) Cohn. También investiga los extractos botánicos, ha empezado a cultivar Tagetes spp. para extraer los ésteres de xantofilas y aplicarlos para el control de nematodos. El laboratorio está orientado a evaluar formulaciones de hongos entomopatógenos, también en la producción de las bacterias que utilizan los nematodos a través de la fermentación y en la eficacia del nematodo para controlar plagas.

\section{Literatura Citada}

Acosta, N.M. y R.D. Cave. 1994. Inventario de los parasitoides de Liriomyza spp. (Diptera: Agromyzidae) en la región sur de Honduras. Revista de Biología Tropical 42: 201-216.

Ávila Sosa, O.A. 2010. Control de Broca del Café (Hypothenemushampei) utilizando once cepas de Beauveria bassiana y el nematodo Heterorhabditis bacteriophora. Tesis Ing. Agr., Escuela Agrícola Panamericana, Zamorano, Honduras

Bográn, C., J.J. Obrycki y R. Cave. 1998. Assessment of biological control of Bemisia tabaci (Homoptera: Aleyrodidae) on common bean in Honduras. Florida Entomologist 81: 384-395.

Cañas Castro, L.A. 1996. Response of natural enemies to sugar applications in maize and their effect on fall armyworm Spodoptera frugiperda (J. E. Smith) populations in Honduras. Tesis MSc, Purdue University, West Lafayette, IN.

Castillo Samudio, R.R. 2007. Efecto de la aplicación de (Trichoderma harzianum) en la producción de maíz dulce (Zea mays) variedad Golden Baby. Tesis Ing. Agr., Escuela Agrícola Panamericana, Zamorano, Honduras.

Cave, R.D. 1992. Inventory of parasitic organisms of the striped grass looper, Mocis latipes (Lepidoptera: Noctuidae), in Honduras. Florida Entomologist 75(4): 592-598.

Cave, R.D. 1993. Parasitoides larvales y pupales de Spodoptera frugiperda (Smith) (Lepidoptera: Noctuidae) en Centro América con una clave para las especies encontradas en Honduras. Ceiba 34: 33-56.

Cave, R.D. 1994. ¿Es viable el control biológico de un vector de geminivirus, como Bemisia tabaci? Manejo Integrado de Plagas (Costa Rica) 34: 18-22. 
Cave, R.D. 1995a. Manual para el Reconocimiento de Parasitoides de Plagas Agrícolas en América Central. Zamorano Academic Press, Zamorano, Honduras.

Cave, R.D. (editor). 1995b. Manual para la Enseñanza del Control Biológico en América Latina. Zamorano Academic Press, Zamorano.

Cave, R.D. 1997. Admontia sp., a potential biological control agent of Metamasius callizona in Florida. Journal of the Bromeliad Society 47: 244-249.

Cave, R.D. 2000. Biology, ecology and use in pest management of Telenomus remus. Biocontrol News \& Information 21(1): $21 \mathrm{~N}-26 \mathrm{~N}$.

Cave, R.D. 2008. Lixadmontia franki Wood and Cave released to control the Mexican bromeliad weevil. Tachinid Times 21: 12-14.

Cave, R.D. y N. M. Acosta. 1999. Telenomus remus: un parasitoide para el control biológico de Spodoptera frugiperda. Ceiba 40(2): 215-227.

Cave, R.D. y R.J. Cordero. 1999. Parasitoides de Leptophobia aripa (Lepidoptera: Pieridae) in Honduras. Ceiba 40(1): 51-55.

Cave, R.D. y G. Márquez C. 1994. Parasitoides de Diaspididae, Coccidae y Aleyrodidae atacando cítricos en Honduras. Ceiba 35: 3-8.

Cherry, A. 1991. Aspects of the interaction between biological and chemical control methods for Plutella xylostella (L.) (Lepidoptera: Yponomeutidae). Disertación Ph.D. University of London, Imperial, College, UK.

Cordero, J. y R.D. Cave. 1992. Natural enemies of Plutella xylostella (LEP.: PLUTELLIDAE) on crucifers in Honduras. Entomophaga 37(3): 397-407.

Cordero, R.J., H.N. Pitre y R.D. Cave. 2000. Influence of weed management and parasitoids on lepidopterous pests in intercropped sorghum and maize in southern Honduras. Ceiba 41(1): 41-52.

Dejud, I., A. Pitty y R.D. Cave. 1992. Control biológico del lirio de agua (Eichhornia crassipes) con Neochetina eichhorniae y N. bruchi. Resumen $4^{\text {to }}$ Congreso Internacional de Manejo Integrado de Plagas, p 49.

Dieguez Gonzales, V.H. 2010. Susceptibilidad del nematodo entomopatógeno (Heterorhabditis bacteriophora) a nueve plaguicidas. Tesis Ing. Agr., Escuela Agrícola Panamericana, Zamorano, Honduras.

Escribano, A., T. Williams, D. Goulson, R.D. Cave, J.W. Chapman y P. Caballero. 1999. Selection of a nucleopolyhedrovirus for control of Spodoptera frugiperda (Lepidoptera: Noctuidae): structural, genetic and biological comparison of four isolates from the Americas. Journal of Economic Entomology 92: 1079-1085.
Espinoza, A. y A. Cherry. 1994. Efecto de la insolación sobre la persistencia del virus de poliedrosis nuclear Galleria mellonella (L.). Ceiba 35: 53-56.

Espinoza, A., A. Cherry y R.D. Cave. 1994. Efecto del VPN Galleria mellonella (L.) sobre larvas de Plutella xylostella (L.). Ceiba 35: 57-61.

Gómez Cabrera, L.E. 1995. Control biológico clásico de Bemisia tabaci (Gennadius) en Honduras. Tesis Ing. Agr., Escuela Agrícola Panamericana, Zamorano, Honduras.

González, A. y R.D. Cave. 1997a. Comparación de las poblaciones de arañas foliares diurnas en frijol común bajo dos sistemas de labranza. Ceiba 38: 47-50.

González, A. y R.D. Cave. 1997b. Comparación del parasitismo de huevos de Empoasca kraemeri Ross \& Moore (Homoptera: Cicadellidae) por Anagrus spp. (Hymenoptera: Mymaridae) en frijol común en labranza cero y labranza convencional. Ceiba 38:51-56.

Holm, L.G., D.L. Plucknett, J.V. Pancho y J.P. Herberger. 1977. The World's Worst Weeds: Distribution and Biology. The University Press of Hawaii, Honolulu, Estados Unidos. 609 p.

Juárez Figueroa, R.H. 2007. Control del escarabajo del estiércol Alphitobius diaperinus con Heterorhabditis bacteriophora, Beauberia bassiana y Metarhizium anisopliae. Tesis Ing. Agr., Escuela Agrícola Panamericana, Zamorano, Honduras.

Julien, M.H. 1992. Biological Control of Weeds, A World Catalogue of Agents and their Target Weeds. Third Edition. C.A.B. International.

Mantilla Compte, J. 2007. Movimiento de Trichoderma harzianum en un suelo de textura media cultivado con pepino (Cucumis sativa), suministrado a una y dos horas en el sistema de riego por goteo en el Zamorano, Honduras. Tesis Ing. Agr., Escuela Agrícola Panamericana, Zamorano, Honduras.

Méndez Martinez, J.A. 2003. Efectos de aplicación de Trichoderma harzianum y Paecilomyces lilacinus en el rendimiento de lechuga orgánica en Zamorano. Tesis Ing. Agr., Escuela Agrícola Panamericana, Zamorano, Honduras.

Méndez, N.A. 2008. Evaluación de ocho cepas de Beauveria bassiana para control de broca del café Hypothenemus hampei. Tesis Ing. Agr., Escuela Agrícola Panamericana, Zamorano, Honduras.

Morán Quintero, N.R. 2007. Evaluación de cuatro cepas de Trichoderma harzianum para el control de Rhizoctonia solani en plántulas de pepino (Cucumis sativa). Tesis Ing. Agr., Escuela Agrícola Panamericana, Zamorano, Honduras. 
Morán Ruiz, F.S. 2007. Efectividad del fraccionamiento de la dosis comercial $3 \times 10^{11} \mathrm{UFC} / \mathrm{ha}$ de Trichozam $^{\circledR}$ (Trichoderma harzianum) en el crecimiento de las plántulas de siete cultivos hortícolas. Tesis Ing. Agr., Escuela Agrícola Panamericana, Zamorano, Honduras.

Njoka, S.W., G.R.S. Ochiel, J.O. Manyalar y J.B. OkeyoOwuor. 2006. The life history and survival of Neochetina in Lake Victoria basin: Basis for biological weed control. In Proceedings of the $11^{\text {th }}$ World Lakes Conference, vol. 2. E. Odada, D.O. Olago, W. Ochola, M. Ntiba, S. Wandiga, N. Gichuki y H. Oyieke, eds. p 593-599.

Morjan Erazo, W.E. 1993. Depredadores nocturnos de plagas de maíz y de frijol en dos sistemas de labranza. Tesis Ing. Agr., Escuela Agrícola Panamericana, Zamorano, Honduras.

Pantoja Guamán, D.O. 2009. Capacidad depredadora de Orius insidiosus (Say) sobre Thrips tabaci (Lindeman) en laboratorio y en cultivo de pepino. Tesis Ing. Agr., Escuela Agrícola Panamericana, Zamorano, Honduras.

Parada López, J.E. 2007. Estudio de factibilidad para la comercialización a través de E-commerce de bioplaguicidad marca Zamorano en Honduras. Tesis Ing. Agr., Escuela Agrícola Panamericana, Zamorano, Honduras.

Perrera Viamil, A.A.2009. Efectividad del nematodo Heterorhabditis bacteriophora para el control de larvas de Phyllophaga spp. Tesis Ing. Agr., Escuela Agrícola Panamericana, Zamorano, Honduras.

Pitty, A. 2004. Control biológico del lirio acuático. La Tribuna, Tegucigalpa (Honduras); agosto 21:7B.

Pitty, A., I. Dejud e I. Zelaya. 1992. El temido lirio de agua es controlado con insectos. Diario El Heraldo, Revista Agropecuaria Zamorano, septiembre 30, año 1, \# 9:26.

Pitty, A., I. Dejud, I. Zelaya y H. Barletta. 1993. Control biológico del lirio acuático. Agricultura de las Américas 42(6):18-20.

Rivera Jerez, C.G. 2004. Evaluación de sensibilidad in vitro de Trichozam (Trichoderma harzianum) a nueve fungicidas. Tesis Ing. Agr., Escuela Agrícola Panamericana, Zamorano, Honduras.

Romero Villagra, D.C. 2004. Efectos de la aplicación de Paecilomyces lilacinus en el control de Meloidogyne spp. en pepino. Tesis Ing. Agr., Escuela Agrícola Panamericana, Zamorano, Honduras.

Rosero Asqui. A. 2008. Evaluación de cuatro cepas de Trichoderma sp. y sus combinaciones para el control de Fusarium sp. en sandía. Tesis Ing. Agr., Escuela Agrícola Panamericana, Zamorano, Honduras.

Raudes Reyes, M.G. 2006. Efecto de la aplicación de Trichozam (Trichoderma harzianum) en la promoción del rendimiento de tomate, chile dulce y pepino en invernaderos de Zamorano. Tesis Ing. Agr., Escuela
Agrícola Panamericana, Zamorano, Honduras.

Suazo López, O.O. 2008. Optimización de los parámetros de aplicación de Heterorhabditis bacteriophora para el control de Spodoptera frugiperda en el cultivo de maíz en condiciones de campo, Zamorano, Honduras. Tesis Ing. Agr., Escuela Agrícola Panamericana, Zamorano, Honduras.

Toapanta Valencia, L.D. 2005. Efecto de la aplicación de Beauveria bassiana y Metarhizium anisopliae para el control de Phyllophaga spp. y Aeolus spp. en cultivo de camote (Ipomoea batatas). Tesis Ing. Agr., Escuela Agrícola Panamericana, Zamorano, Honduras.

Turcios Rivas, E.E. 2009. Evaluación del movimiento del nematodo Heterorhabditis bacteriophora y su capacidad infectiva bajo condiciones controladas de humedad y tres texturas de suelo. Tesis Ing. Agr., Escuela Agrícola Panamericana, Zamorano, Honduras.

Turcios Rivera, C.W. 2009. Preferencia de depredación de Neoseiulus californicus (McGregor) (Acari: Phytoseiidae) sobre huevos, ninfas y adultos de Tetranychus gloveri (Banks) y T. ludeni (Zacher) (Acari: Tetranychidae) en Zamorano, Honduras. Tesis Ing. Agr., Escuela Agrícola Panamericana, Zamorano, Honduras.

Vaquedano Gámez, L.C. 2006. Efecto de la aplicación de hongos entomopatógenos para el control de plagas en el cultivo de pepino, en el valle de Comayagua, Honduras. Tesis Ing. Agr., Escuela Agrícola Panamericana, Zamorano, Honduras.

Venter, N., M.P. Hill, S.L. Hutchinson, B.S. Ripley. 2013. Weevil borne microbes contribute as much to the reduction of photosynthesis in water hyacinth as does herbivory. Biological Control 64:138-142

Villarroel Basantes, R.I. 2009. Respuesta funcional, respuesta numérica e interferencia de Neoseiulus californicus (McGregor) (Acari: Phytoseiidae) sobre Tetranychus ludeni (Zacher) y Tetranychus gloveri (Banks) (Acari: Tetranychidae) en Zamorano, Honduras. Tesis Ing. Agr., Escuela Agrícola Panamericana, Zamorano, Honduras.

Williams, T., D. Goulson, P. Caballero, J. Cisneros, A.M. Martínez, J.W. Chapman, D. X. Roman y R.D. Cave. 1999. Evaluation of a baculovirus bioinsecticide for small-scale maize growers in Latin America. Biological Control 14: 67-75.

Wood, D.M. y R.D. Cave. 2006. Description of a new genus and species of weevil parasitoid from Honduras (Diptera: Tachinidae). Florida Entomologist 89:239244.

Recibido para publicación el 19 de julio del 2012. Aceptado para publicación el 22 de octubre de 2012. 